\title{
Double Field Theory description of heterotic gauge symmetry enhancing-breaking
}

\author{
G. Aldazabal,,$^{a, b}$ E. Andrés, ${ }^{b}$ M. Mayo ${ }^{a, b}$ and V. Penas ${ }^{a, b}$ \\ ${ }^{a}$ G. Física CAB-CNEA and CONICET, Centro Atómico Bariloche, \\ Av. Bustillo 9500, Bariloche, Argentina \\ ${ }^{b}$ Instituto Balseiro, \\ Av. Bustillo 9500, Bariloche, Argentina \\ E-mail: aldazaba@cab.cnea.gov.ar, andres@cab.cnea.gov.ar, \\ martin.mayo@ib.edu.ar, victor.penas@cab.cnea.gov.ar
}

Abstract: A Double Field Theory (DFT) description of gauge symmetry enhancingbreaking in the heterotic string is presented. The construction, based on previous results for the bosonic string, relies on the extension of the tangent frame of DFT. The fluxes of a Scherk-Schwarz like generalized toroidal compactification are moduli dependent and become identified with the structure constants of the enhanced group at fixed "self-dual" points in moduli space. Slight displacements from such points provide the breaking of the symmetry, gauge bosons acquiring masses proportional to fluxes. The inclusion of fermions is also discussed.

KeYwOrds: Flux compactifications, String Duality, Supergravity Models, Superstrings and Heterotic Strings

ARXIV EPRINT: 1708.07148 


\section{Contents}

1 Introduction 1

2 Heterotic gauge symmetry enhancement and DFT rewriting 2

2.1 Gauge symmetry breaking from DFT rewriting 5

$\begin{array}{lll}2.1 .1 & \text { Goldstone bosons } & 7\end{array}$

2.1.2 Vector masses 8

2.1.3 Scalar masses $\quad 8$

2.2 Examples 9

$2.2 .1 \quad \mathrm{SU}(2) \times \mathrm{SO}(32) \times \mathrm{U}(1) \quad 9$

$\begin{array}{lll}2.2 .2 & \mathrm{SO}(34) & 10\end{array}$

$\begin{array}{llr}3 & \text { Including fermions } & 10\end{array}$

4 Summary and outlook $\quad 12$

$\begin{array}{lr}\text { A Heterotic DFT } & 14\end{array}$

$\begin{array}{ll}\text { B Some heterotic string basics } & 17\end{array}$

\section{Introduction}

The possibility of understanding gauge symmetry enhancement from a Double Field Theory (DFT) perspective was addressed in various recent articles [1-3]. The discussion was done in the context of the bosonic string since, even if ill defined, it is the simplest example in several aspects and allows to identify the relevant ingredients. In the present note we follow similar steps as in [3] in order to describe the gauge symmetry enhancement (and breaking) in the heterotic string from a DFT-like formulation.

Gauge symmetry enhancement is a very stringy phenomena associated to the fact that the string is an extended object and, therefore, it can wind around non-contractible cycles. String states are thus characterized by a stringy quantum number, the so-called winding number, counting the number of times that the cycle is wrapped by the string. The exchange of winding and momentum states (accompanied by a transformation of moduli fields) leads to T-duality invariance, a genuine stringy feature.

At certain moduli points (fixed points of T-duality transformations) vector boson states in some combinations of windings and momenta become massless and give rise to enhanced gauge symmetries (see for instance [4-6]). Of course, the effective low energy theory, where massive states are neglected, can be described by an usual gauge field theory Lagrangian, containing gravity, with no reference to any windings. An intriguing aspect is that this 
field theory somehow encodes information about stringy effects. Moreover, even if gauge symmetry breaking is achieved as usual, with some scalar fields acquiring vevs, this higgsing process must encode information about moduli away from the fixed point.

Interestingly enough, this effective theory close to self-dual points originated in the bosonic string, can be embedded [3] into a DFT-like formulation. In DFT (we will be more precise below) the internal configuration space includes, besides the usual space coordinates dual to KK momenta, new coordinates dual to winding states and therefore, coordinates are doubled. This DFT rewriting allows to highlight the stringy aspects of these gauge theories. Actually, in a generalized Scherk-Schwarz [7-9] compactification of this DFT the fluxes, computed from an internal vielbein depending on doubled coordinates, appear to depend on moduli and become the structure constants of the enhanced group at fixed points. We show below that this rewriting also works for the bosonic sector of a toroidally compactified heterotic string. Moreover, we show that by invoking supersymmetry, a corresponding fermionic sector can also be introduced.

In section 2 we present a brief discussion of symmetry enhancement and show the DFT rewriting of heterotic string theory effective action close or at the enhancing points. It is also shown how breaking of gauge symmetry is encoded into the moduli dependence of fluxes. A simple illustration for the case of circle compactification is provided. Ideas presented in [3] are recurrently used throughout the article.

The introduction of fermions is discussed in section 3. In particular we show that if the gaugings in shift matrices of gauged supergravities, associated to fermionic mass terms, are replaced by Scherk-Schwarz (moduli dependent) fluxes, the masses of fermions are in correspondence with their bosonic partners, as expected from supersymmetry.

Several details are presented in the appendices. In appendix A a quick introduction to DFT and generalized Scherk-Schwarz like compactification is provided with emphasis in the heterotic case where the ingredients needed in our construction are highlighted. For a more complete introduction to DFT we provide some of the original references in [10-14] and refer the reader to some reviews [15-17] (where a more extensive list of references can be found). In appendix B a brief account of heterotic string features needed for our discussion is presented.

Concluding remarks and a brief outlook are presented in section 4 .

\section{Heterotic gauge symmetry enhancement and DFT rewriting}

Toroidal compactification of the $\mathrm{SO}(32)$ (or $E_{8} \times E_{8}$ ) heterotic string to $d$ space-time dimensions leads to a generic gauge group

$$
G_{L} \times \mathrm{U}(1)_{R}^{10-d}
$$

where the Left group $G_{L}$ is generically a product of non-abelian and abelian gauge groups. The rank of $G_{L}$ is $r_{L}=16+10-d=26-d$ originated from the 16 Cartan generators of the ten dimensional gauge group plus the $r=10-d$ vector bosons coming from left combinations of the KK reductions of the metric and the antisymmetric tensor. Different gauge groups do appear when moving along moduli space. At generic points in moduli 
space $G_{L}=\mathrm{U}(1)_{L}^{26-d}$ while a point of maximum enhancement leads to $G_{L}=\mathrm{SO}(52-2 d)$ for the $\mathrm{SO}(32)$ string case.

We present some basic details in appendix B. Let $n=n_{c}+r_{L}=\operatorname{dim} G_{L}$ be the dimension of $G_{L}$ at some moduli point with $n_{c}$ denoting the number of charged generators. The effective low energy theory will thus be a $G_{L} \times \mathrm{U}(1)_{R}^{10-d}$ gauge theory coupled to gravity and the Kalb-Ramond antisymmetric tensor field in $d$ dimensions. There are also $\left(n_{c}+26-d\right)(10-d)$ scalars. Thus, the counting of degrees of freedom leads to: $d^{2}$ corresponding to graviton plus B field, $n_{c}+36-2 d$ vectors from $G_{L} \times \mathrm{U}(1)_{R}^{10-d}$ and $\left(n_{c}+26-d\right)(10-d)$ scalars. Recall that the number of scalar fields corresponds to $(26-d)(10-d)$ moduli plus $n_{c}(10-d)$ extra scalars that should become massive at generic points where the broken gauge group is $\mathrm{U}(1)_{L}^{26-d} \times \mathrm{U}(1)_{R}^{10-d}$.

It is interesting to notice that the total number of degrees of freedom coincides with

$$
\operatorname{dim} \frac{O(d+n, d+r)}{O(d+n) \times O(d+r)}=d^{2}+d\left(n_{c}+36-2 d\right)+\left(n_{c}+26-d\right)(10-d) .
$$

Indeed, this coset-like writing provides a clue of how to express the effective theory in a DFT-like form as discussed in appendix A.

Following similar steps as presented in $[1,3]$ for the bosonic string case, we propose an expression for such an action and then discuss its specific features. Namely,

$$
\begin{aligned}
S_{\text {eff }}= & \frac{1}{2 \kappa_{d}^{2}} \int d^{d} x \sqrt{g} e^{-2 \varphi}\left[\mathcal{R}+4 \partial^{\mu} \varphi \partial_{\mu} \varphi-\frac{1}{12} H_{\mu \nu \rho} H^{\mu \nu \rho}\right. \\
& \left.-\frac{1}{8} \mathcal{H}_{A B} F^{A \mu \nu} F_{\mu \nu}^{B}+\frac{1}{8}\left(D_{\mu} \mathcal{H}\right)_{A B}\left(D^{\mu} \mathcal{H}\right)^{A B}-V\right] .
\end{aligned}
$$

Here

$$
V=-\frac{1}{12} f_{A B}{ }^{K} f_{L C}{ }^{D}\left(\mathcal{H}^{A L} \mathcal{H}^{B C} \mathcal{H}_{K D}-3 \mathcal{H}^{A L} \eta^{B C} \eta_{K D}+2 \eta^{A L} \eta^{B C} \eta_{K D}\right)-\Lambda
$$

is a scalar potential where the last two terms are just constants. The scalars parametrize the coset $\frac{O(n, r)}{O(n) \times O(r)}$ of dimension $\left(n_{c}+26-d\right)(10-d)$. The indices can be conveniently split in a L-R basis (named a $\mathcal{C}$ base) as $A=(a, \hat{I})$ where $a=1, \ldots r_{L}, r_{L}+1, \ldots r_{L}+n_{c}=n=\operatorname{dim} G_{L}$ index runs over the Left group $G_{L}$. In addition the $\hat{I}=1, \ldots r$ index corresponds to the Right $\mathrm{U}(1)^{r}$ group. The index contractions are performed with $\eta^{A B}$, the $O\left(r_{L}+n_{c}, r\right)$ invariant metric

$$
\eta^{A B}=\left(\begin{array}{cc}
1_{r_{L}+n_{c}} & 0 \\
0 & -1_{r}
\end{array}\right) .
$$

$\mathcal{H}_{A B}$ is the (so-called) internal generalized metric encoding information about scalar fields. $\mathcal{R}$ is the $d$-dimensional Ricci scalar and $F_{\mu \nu}^{A}$ and $H_{\mu \nu \rho}$

$$
\begin{aligned}
F^{B} & =d A^{B}-\frac{1}{2 \sqrt{2}} f_{C D}{ }^{B} A^{C} \wedge A^{D} \\
H & =d B+F^{C} \wedge A_{C}-\frac{1}{3 ! \sqrt{2}} f_{A B C} A^{A} \wedge A^{B} \wedge A^{C},
\end{aligned}
$$

are the gauge field and $B$ field strengths. 
The covariant derivative of the scalars is

$$
\left(D_{\mu} \mathcal{H}\right)_{A B}=\left(\partial_{\mu} \mathcal{H}\right)_{A B}+\frac{1}{\sqrt{2}} f^{K}{ }_{L A} A_{\mu}^{L} \mathcal{H}_{K B}+\frac{1}{\sqrt{2}} f^{K}{ }_{L B} A_{\mu}^{L} \mathcal{H}_{A K} .
$$

Finally, the $f_{A B C}=\eta_{A K} f^{K}{ }_{B C}$ are completely antisymmetric constants. Interestingly enough this action can be interpreted as a generalized Scherk-Schwarz reduction of a DFTlike action, as we briefly sketch in appendix A, the constants $f^{K}{ }_{B C}$ being the generalized fluxes of the compactification. ${ }^{1}$ There are $\frac{(r+n)(r-1+n)(r-2+n)}{3 !}$ such fluxes which must satisfy the quadratic constraints

$$
f_{[A B}{ }^{K} f_{C D] K}=0
$$

If indices are allowed to transform then the action is globally invariant under $O\left(n_{c}+26-d, 10-d\right)$ and it can be identified with the bosonic (electric) sector of a halfmaximal gauged supergravity action [18-21].

In spite of the fact that this huge number of gaugings was explored in several situations, its physical interpretation deserves further investigation. For instance, if we restricted to $a=1, \ldots, r=10-d$, and in $r=6$ dimensions, the above counting of fluxes would correspond to the 220 gaugings of electric sector of $O(6,6)$ gauged supergravity. These gaugings have been identified (see for instance [15, 19, 22-24]) as geometric and non geometric fluxes in (orientifold) string compactifications. Here we will restrict to a very specific choice of a subset of all possible fluxes, relevant to our discussion.

In order to make contact with the heterotic string effective action we first expand the generalized metric in terms of scalar fluctuations encoded in the scalar matrix $M_{a, \hat{I}}$ with $\operatorname{dim} G_{L} \times r=\left(n_{c}+26-d\right)(10-d)$ independent degrees of freedom. Namely we write

$$
\mathcal{H}_{\mathcal{C}}^{A B}=\delta^{A B}+\mathcal{H}^{(1) A B}+\frac{1}{2} \mathcal{H}^{(2) A B}+\ldots
$$

such that matrix elements vanish unless

$$
\begin{array}{ll}
\mathcal{H}_{a \hat{I}}^{(1)}=M_{a, \hat{I}}, & \mathcal{H}_{\hat{I} a}^{(1)}=M_{a, \hat{I}}^{T} \\
\mathcal{H}_{a b}^{(2)}=\left(M M^{T}\right)_{a b}, & \mathcal{H}_{\hat{I} \hat{J}}^{(2)}=\left(M^{T} M\right)_{\hat{I} \hat{J}} .
\end{array}
$$

Moreover, we make a specific choice for flux values (therefore breaking the global symmetry), by identifying them with the gauge group structure constants. Namely,

$$
f_{A B C}= \begin{cases}f_{a b c} & G_{L} \text { structure constants } \\ 0 & \text { otherwise }\end{cases}
$$

where $f_{a b c}$ is the subset of all possible fluxes (with Left indices) reproducing the structure constants of the $G_{L}$ group algebra. When couplings are adequately adjusted the above

\footnotetext{
${ }^{1}$ Recall that other kinds of fluxes like $f_{A}$ could be present $[8,9,15,34]$ as shown in appendix A. Here we set them to zero since they are not relevant for our discussion.
} 
action reduces to the $G_{L} \times \mathrm{U}(1)_{R}^{10-d}$ gauge theory action

$$
\begin{aligned}
S= & \frac{1}{2 \kappa_{d}^{2}} \int d^{d} x \sqrt{g} e^{-2 \varphi}\left(\mathcal{R}+4 \partial^{\mu} \varphi \partial_{\mu} \varphi-\frac{1}{12} H_{\mu \nu \rho} H^{\mu \nu \rho}\right) \\
& -\frac{1}{8}\left(\delta_{a b} F^{a \mu \nu} F_{\mu \nu}^{b}+\delta_{\hat{I} \hat{J}} \bar{F}^{\hat{I} \mu \nu} \bar{F}_{\mu \nu}^{\hat{I}}-\frac{1}{2} g_{d} \sqrt{\alpha^{\prime}} M_{a \hat{I}} F_{\mu \nu}^{a} \bar{F}^{\hat{I} \mu \nu}\right) \\
& -D_{\mu} M_{a \hat{I}} D_{\nu} M^{a \hat{I}} g^{\mu \nu}+\mathcal{O}\left(M^{4}\right),
\end{aligned}
$$

reproducing the bosonic sector of heterotic string low energy theory at a fixed point. Here $a$ labels the Left gauge group (generically non- Abelian) generators with vector bosons $A_{L \mu}^{a}$ and $\hat{I}=1, \ldots r$ the Abelian group $\mathrm{U}(1)_{\hat{I}}$ associated to vector bosons $A_{R \mu}^{\hat{I}}$. The scalar fields live in the $\left(\mathbf{d i m G}_{\mathbf{L}}\right)_{\hat{\mathbf{q}}=\mathbf{0}}$ adjoint representation of $G_{L}$ and carry zero vector charge $\hat{\mathbf{q}}=\left(\hat{q}_{1}, \ldots, \hat{q}_{r}\right)=0$ with respect to $\mathrm{U}(1)_{R}^{r}$ right group. Thus, the covariant derivative in (2.7) becomes

$$
D_{\mu} M_{a \hat{I}}=\partial_{\mu} M_{a \hat{I}}+g_{d} f^{k}{ }_{l a} A_{L \mu}^{l} M_{k \hat{I}},
$$

where $g_{d}=\kappa_{d} \sqrt{\frac{2}{\alpha^{\prime}}}$. Notice that no scalar potential is generated for this choice of structure constants.

In the next section we show, in the context of DFT, how gauge symmetry breaking can be achieved by allowing structure constants to depend on moduli, as expected from string theory.

\subsection{Gauge symmetry breaking from DFT rewriting}

In string theory the structure constants can be read out from 3-point vertex vector boson operators. For the Cartan generators, which we label with the index $\check{I}_{L}=(i=1, \ldots r$; $I=1, \ldots 16)$, the associated Left vector bosons vertex operators are of the form $V\left(\check{I}_{L}\right) \propto \partial_{z} y^{\check{I}} \tilde{\psi}^{\mu} e^{i K . X}$, whereas for charged operators we have $V\left(l_{L}\right) \propto \tilde{\psi}^{\mu} e^{i l_{L} \cdot y(z)} e^{i K . X}$ where $l_{L}^{\check{I}}$ are the Left internal momenta defined in (B.2), and $X^{\mu}(z)$ and $K^{\mu}$ are the spacetime coordinate and momentum, respectively. Recall that the internal momenta depend on specific values of KK momenta $p_{m}$ and winding numbers $\tilde{p}^{m}$ as well as on the $\Lambda_{16}$ weights ${ }^{2}$ $P^{I}$. We encode these values into a "generalized momentum vector"

$$
\check{\mathbb{P}}=\left(\mathbb{P} ; P^{I}\right), \quad \text { with } \quad \mathbb{P}=\left(p_{m}, \tilde{p}^{m}\right) .
$$

Let us encode denote by $\Phi=(g, B, A)$ a generic moduli point. Since momenta depend on moduli fields we actually have $l_{L}=l_{L}^{\check{\mathbb{P}}}(\Phi)$ and similarly, $l_{R}=k_{R}^{\check{\mathbb{P}}}(\Phi)$.

At specific points $\Phi_{0}$ in moduli space and for certain values of $\check{\mathbb{P}}$ such that

$$
k_{R}^{\check{\mathbb{P}}}\left(\Phi_{0}\right)=0, \quad\left(l_{L}^{\check{\mathbb{P}}}\left(\Phi_{0}\right)\right)^{2}=2
$$

gauge symmetry enhancement occurs (B.5). At these points

$$
l_{L}^{(\check{\mathbb{P}})}\left(\Phi_{0}\right) \equiv \alpha^{(\check{\mathbb{P}})}
$$

\footnotetext{
${ }^{2}$ For the sake of clarity we concentrate in the $\mathrm{SO}(32)$ string but same conclusions are valid for the $E_{8} \times E_{8}$ heterotic case with lattice $\Lambda_{8} \times \Lambda_{8}$.
} 
become the roots $\alpha^{(\check{\mathbb{P}})}$ of the $G_{L}$ gauge group. Notice that there is an associated root to each of the $n_{c}$ possible values of $\check{\mathbb{P}}$, satisfying the massless vector condition (2.14).

Three point amplitudes involving Left vector boson vertices can be expressed as

$$
\left\langle V_{L}\left(l_{L}^{\left(\check{\mathbb{P}}_{1}\right)}\right) V_{L}\left(l_{L}^{\left(\check{\mathbb{P}}_{2}\right)}\right) V_{L}\left(l_{L}^{\left(\check{\mathbb{P}}_{3}\right)}\right)\right\rangle \propto f_{\alpha^{\left(\check{\mathbb{P}}_{1}\right)} \alpha^{\left(\check{\mathbb{P}}_{2}\right)} \alpha^{\left(\check{\mathbb{P}}_{3}\right)}(\Phi)} E\left(\epsilon_{1}, K_{1} ; \epsilon_{2}, K_{2} ; \epsilon_{3}, K_{3}\right),
$$

where $E\left(\epsilon_{i}, K_{i} ; i=1,2,3\right)$ is a Lorentz invariant antisymmetric function of polarization

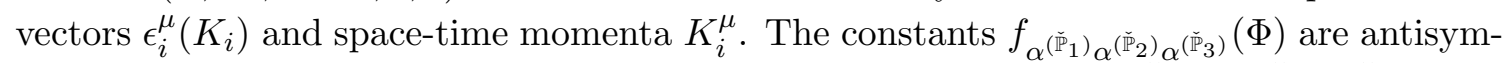
metric and vanish unless internal momentum is conserved, namely $\check{\mathbb{P}}_{3}=-\check{\mathbb{P}}_{1}-\check{\mathbb{P}}_{2}$. At a self-dual point $\Phi=\Phi_{0}$ this indicates that structure constants $f_{\alpha_{1} \alpha_{2} \alpha_{3}}$ vanish unless $\alpha_{1}+\alpha_{2}$

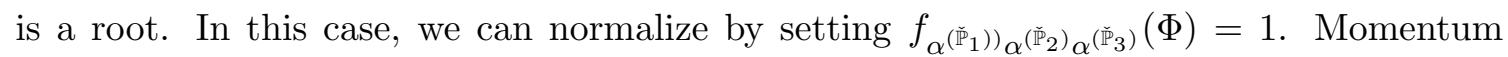
conservation also implies that, at the self-dual point, amplitudes mixing Left and Right indices vanish. However, away from the fixed point, the vertices develop a dependence on $l_{R}, V_{L}\left(l=\left(l_{L}, l_{R}\right)\right) \propto e^{i l_{L}^{\tilde{\tilde{\mu}}}(\Phi) \cdot y(z)+l_{R}^{\tilde{\tilde{m}}}(\Phi) \cdot \bar{y}(\bar{z})} e^{i K \cdot X}$ and therefore mixing now occurs. In fact, it is found that the only non vanishing amplitudes are

$$
\left\langle V_{L}\left(l^{(\check{\mathbb{P}})}\right) V_{L}\left(l^{(-\check{\mathbb{P}})}\right) V_{L}(\check{I})\right\rangle \propto l_{L}^{(\check{\mathbb{P}})}(\Phi)_{\check{I}} ; \quad\left\langle V_{L}\left(l^{(\check{\mathbb{P}})}\right) V_{L}\left(l^{(-\check{\mathbb{P}})}\right) V_{R}(\hat{I})\right\rangle \propto l_{R}^{(\check{\mathbb{P}})}(\Phi)_{\hat{I}}
$$

Following [3], we propose to identify the amplitude coefficients with some algebra structure constants, even (slightly) away from the fixed point $\Phi_{0}$. Namely we set

$$
f_{\alpha^{(\check{P})} \alpha^{(-\check{P})} \check{I}_{L}}(\Phi)=l_{L}^{(\mathbb{P})}(\Phi)_{\check{I}}, \quad f_{\alpha^{(\mathbb{P})} \alpha^{(-\check{P})} \hat{I}}(\Phi)=l_{R}^{(\check{\mathbb{P}})}(\Phi)_{\hat{I}}
$$

with the other constants being obtained as permutations, and we propose the algebra

$$
\begin{array}{rlrl}
{\left[E_{\alpha}, E_{-\alpha}\right]} & =l_{L}^{(\alpha) \check{I}} H_{\check{I}}+l_{R}^{(\alpha) \hat{I}} \hat{H}_{\hat{I}} & & {\left[H_{I}, E_{\alpha}\right]=l_{L}^{(\alpha) I} E_{\alpha}} \\
{\left[E_{\alpha_{1}}, E_{\alpha_{2}}\right]=f_{\alpha_{1} \alpha_{2} \alpha_{3}} E_{\alpha_{3}}} & & {\left[\hat{H}_{I}, E_{\alpha}\right]=l_{R}^{(\alpha) I} E_{\alpha} .}
\end{array}
$$

We have used $\alpha=\alpha^{(\mathbb{P})}$ to alleviate the notation and, as we found above, $f_{\alpha_{1} \alpha_{2} \alpha_{3}}=1$ if $\alpha_{3}=\alpha_{1}+\alpha_{2}$ is a root and vanishes otherwise. All other commutators vanish. It is easy to show that (2.18) satisfies Jacobi identities and, therefore, defines a Lie algebra.

Recall that, at the self-dual point where $k_{R}^{\alpha}\left(\Phi_{0}\right)=0$ and $f_{\alpha-\alpha \hat{I}}=l_{L}^{(\alpha) \hat{I}}=\alpha^{\hat{I}}$, the algebra reduces to the gauge algebra of $G_{L}$ group in the Cartan-Weyl basis. For instance $\left[E_{\alpha}, E_{-\alpha}\right]=\alpha^{\hat{I}} H_{\hat{I}}$ for charged generators $E_{\alpha}$ and Cartan generators $H_{\hat{I}}$, as expected. Interestingly enough, by performing a linear combination of generators it can be shown that there is still an underlying $G_{L}$ algebra. To visualize the linear combination let us define a double Cartan operator $\mathbb{H}_{A}=\left(H_{\check{I}}, \hat{H}_{\hat{I}}\right)$ and the double (moduli dependent) momentum $\mathbb{L}_{A}^{(\alpha)}=\left(l_{L}^{(\alpha) \check{I}}, l_{R}^{(\alpha) I}\right)$. The algebra (2.18) can now be written as

$$
\begin{aligned}
{\left[E_{\alpha}, E_{-\alpha}\right] } & =\mathbb{L}_{A}^{(\alpha)} \mathbb{H}_{A} \\
{\left[\mathbb{H}_{A}, E_{\alpha}\right] } & =\mathbb{L}_{A}^{(\alpha)} E_{\alpha} \\
{\left[E_{\alpha_{1}}, E_{\alpha_{2}}\right] } & =f_{\alpha_{1} \alpha_{2} \alpha_{3}} E_{\alpha_{3}} .
\end{aligned}
$$


It is worth observing that an $O\left(r_{L}, r_{R}\right)$ transformation can be performed over the double Cartan generator, namely the one given by the inverse of

$$
\left(\begin{array}{ccc}
\delta_{I J} & 0 & A_{J n} \delta_{n m} \\
-A_{m}^{J} \delta_{J I} & \delta_{n m} & G_{n m}-B_{n m}-\frac{R}{2} A_{n}^{I} A_{m}^{I} \\
-A_{m}^{J} \delta_{J I} & \delta_{n m} & -G_{n m}-B_{n m}-\frac{R}{2} A_{n}^{I} A_{m}^{I}
\end{array}\right)
$$

such that $\mathbb{L}^{(\alpha)}$ is mapped to $\check{\mathbb{P}}$ (see $\left.(2.13)\right)$ and $\mathbb{H}$ to new double Cartan's $\mathcal{H}$ leading to

$$
\begin{aligned}
{\left[E_{\alpha}, E_{-\alpha}\right] } & =\check{\mathbb{P}}_{A}^{(\alpha)} \mathcal{H}_{A} \\
{\left[\mathcal{H}_{A}, E_{\alpha}\right] } & =\check{\mathbb{P}}_{A}^{(\alpha)} E_{\alpha} \\
{\left[E_{\alpha_{1}}, E_{\alpha_{2}}\right] } & =f_{\alpha_{1} \alpha_{2} \alpha_{3}} E_{\alpha_{3}} .
\end{aligned}
$$

This final algebra has the same form independently of moduli values. Furthermore since the algebra (2.18) and (2.21) are isomorphic, due to (2.20), we conclude that the algebra at the self-dual point is the same at all other (neighborhood) points.

In generalized Scherk-Schwarz like compactifications of DFT, the generalized fluxes $f_{A B C}$ are defined from the generalized algebra satisfied by the internal frame (A.13). Let us assume for the moment that a specific choice of frame exists such that these fluxes are the structure constants found in (2.18). Once these fluxes are identified we must replace them into the action (2.3). The output is that the resulting action is the gauge broken symmetry action where vector bosons and scalars acquire masses proportional to structure constants mixing Left and Right indices, namely $f_{\alpha^{(\mathbb{P})} \alpha^{(-\mathbb{P})} \hat{I}}(\Phi)$.

\subsubsection{Goldstone bosons}

We start by inspecting the couplings between vectors and scalars arising from kinetic terms in (2.3). By keeping the first term in the internal metric expansion (2.9), $\mathcal{H}_{\mathcal{C}}^{A B}=\delta^{A B}+\mathcal{H}^{(1) A B}+\ldots$ we find that

$$
\begin{aligned}
D_{\mu} \mathcal{H}_{A B} D^{\mu} \mathcal{H}^{A B} & \approx 4 \partial_{\mu} M^{A B} f^{K}{ }_{L A} \delta_{K B} A^{\mu L}=4 \partial_{\mu} M^{a \hat{I}} f_{a L \hat{I}} A^{\mu L} \\
& =4 \partial_{\mu} M^{a \hat{I}} f_{a b \hat{I}} A^{\mu b} .
\end{aligned}
$$

Here we have used the exapnsion into Left and Right indices $A=(a, \hat{I})$, we have used the metric (2.5), the antisymmetry of $f_{A B C}$ and the fact that the only non vanishing fluxes are of the form $f_{a b c}, f_{a b \hat{I}}$.

The conclusion is that, for a given vector boson $A_{\mu}^{b}$, there is a combination of $\hat{I}=1, \ldots, r=10-d$, would-be Goldstone bosons scalar fields $f_{a b \hat{I}} M^{a \hat{I}} \equiv f_{\alpha-\alpha \hat{I}} M^{\alpha \hat{I}}=$ $l_{R}^{(\mathbb{P})}(\Phi)_{\hat{I}} M^{\alpha \hat{I}}$ (whenever $f_{a b \hat{I}} \neq 0$ ). We have recast the expression in a Cartan-Weyl basis by recalling that the only non vanishing fluxes (away from the point of enhancement) containing a Cartan index are of the form $f_{\alpha-\alpha \hat{I}}$.

Interestingly enough, this combination arises as a conformal anomaly contribution in the OPE of energy momentum tensor with scalars whenever these scalars become massive, away from the fixed point (see [1] for a bosonic string example). This indicates that the 
combination $l_{R}^{(\mathbb{P})}(\Phi)_{\hat{I}} M^{\alpha \hat{I}}(K)$ of internal R-momentum and scalar polarizations, must be set to zero. ${ }^{3}$

Let us see, as an example, how vector bosons and scalar masses arise.

\subsubsection{Vector masses}

In order to read the vector boson masses we must just look at quadratic terms in the scalar kinetic term. Thus, following similar steps as above but now keeping just the constant term in the internal metric expansion $(2.9) \mathcal{H}_{A B}=\delta_{A B}+\ldots$ we find

$$
\begin{aligned}
\frac{1}{8}\left(D_{\mu} \mathcal{H}\right)_{A B}\left(D^{\mu} \mathcal{H}\right)^{A B} & \approx \frac{1}{8}\left(f_{R L B} \delta_{K A}+f_{R L A} \delta_{K B}\right) \eta^{R K}\left(f^{P S A} \delta^{P^{\prime} B}+f^{P S B} \delta^{P^{\prime} A}\right) \eta_{P P^{\prime}} A_{\mu}^{L} A_{S}^{\mu} \\
& =2 \frac{1}{8}\left(f_{R L B} f^{P S A} \delta^{P^{\prime} B}+f_{R L B} f^{P S B} \delta^{P^{\prime} A}\right) \delta_{K A} \eta_{P P^{\prime}} \eta^{R K} A_{\mu}^{L} A_{S}^{\mu} \\
& =-\frac{1}{2} f_{\hat{I} a L} f^{\hat{I} a S} A_{\mu}^{L} A_{S}^{\mu}=-\left(f_{\alpha-\alpha \hat{I}}(\Phi)\right)^{2}\left|A^{\alpha}\right|^{2}
\end{aligned}
$$

where, again, a Cartan-Weyl rewriting was used in the last term. Namely, away from the fixed point, the vector bosons acquire a mass $m_{A^{\alpha}}$ given by

$$
m_{A^{\alpha}}^{2}=\sum_{\hat{I}}\left(f_{\alpha-\alpha \hat{I}}(\Phi)\right)^{2}=l_{L}^{2}(\Phi) .
$$

\subsubsection{Scalar masses}

From a DFT point of view, the scalar masses arise from quadratic terms in scalar fluctuations in the scalar potential. Thus, by inserting the expansion (2.9) into the scalar potential (2.4) we find:

$$
-\frac{1}{12} f_{A B C} f_{D E F} \mathcal{H}^{(1) A D} \mathcal{H}^{(1) B E} \delta^{C F}-\frac{1}{12} f_{A B C} f_{D E F} \mathcal{H}^{(2) A D}\left(3 \delta^{B E} \delta^{C F}-3 \eta^{B E} \eta^{C F}\right) .
$$

We notice that, due to the relative minus sign between Left and Right indices in $\eta^{A B}$ (see $(2.5))$ the second term vanishes unless indices organize as $\delta^{b e} \delta^{\hat{I} \hat{J}}$ leading to

$$
\frac{1}{2} \sum_{\alpha, \hat{I}} f_{\alpha-\alpha \hat{I}} f_{\alpha-\alpha \hat{I}} \mathcal{H}^{(2) \alpha \alpha}=\frac{1}{4} \sum_{\alpha, \hat{I}, \hat{J}}\left(f_{\alpha-\alpha \hat{I}}\right)^{2}\left|M^{\alpha \hat{J}}\right|^{2}=\frac{1}{4} \sum_{\alpha, \hat{J}} m_{\alpha}^{2}\left|M^{\alpha \hat{J}}\right|^{2},
$$

where

$$
m_{\alpha}^{2}=\sum_{\hat{I}}\left(f_{\alpha-\alpha \hat{I}}(\Phi)\right)^{2}=m_{A^{\alpha}}^{2}
$$

is the mass (square) of the scalar field $M^{\alpha \hat{J}}$, coinciding with the vector boson mass.

On the other hand, the first term contribution in (2.26) leads to

$$
\frac{1}{2} \sum_{\alpha}\left(\sum_{\hat{I}} f_{\alpha-\alpha \hat{I}} M^{\alpha \hat{I}}\right)^{2}
$$

\footnotetext{
${ }^{3}$ Alternatively, such a combination of scalar vertex operators must be included into a new massive, anomaly free, vector field.
} 
However, this contribution is irrelevant since $\left(\sum_{\hat{I}} f_{\alpha-\alpha \hat{I}^{M}} M^{\alpha \hat{I}}\right)$ is the Goldstone boson combination.

Let us stress that the obtained masses coincide with the masses computed from string mass formula (B.3).

\subsection{Examples}

Here we discuss a simple illustration of the above construction in the simplest case of compactification on a circle of radius $R$. In this case (B.2) reads

$$
\begin{aligned}
K_{L}^{I} & =P^{I}+R A^{I} \tilde{p} \\
k_{L} & =\sqrt{\frac{\alpha^{\prime}}{2}}\left[\frac{p}{R}+\frac{\tilde{p}}{\tilde{R}}-P . A-\frac{R}{2} A . A \tilde{p}\right] \\
k_{R} & =\sqrt{\frac{\alpha^{\prime}}{2}}\left[\frac{p}{R}-\frac{\tilde{p}}{\tilde{R}}-P . A-\frac{R}{2} A . A \tilde{p}\right] .
\end{aligned}
$$

A massless state requires $k_{R}=0\left(\bar{N}_{F}=1\right)$ and then $k_{L}=\sqrt{2 \alpha^{\prime}} \frac{\tilde{p}}{\tilde{R}}$.

\subsection{1 $\quad \mathrm{SU}(2) \times \mathrm{SO}(32) \times \mathrm{U}(1)$}

A possible set of massless vectors is provided by choosing $p=\tilde{p}= \pm 1$, by setting the radius to its self-dual value $R=\sqrt{\alpha^{\prime}}=\tilde{R}$ and $A^{I}=0$. Together with the massless vector state associated to KK compactification (with $p=\tilde{p}=0=P^{I}$ ) mode these massless vectors lead to an $\mathrm{SU}(2)$ Left group.

In addition, an $\mathrm{SO}(32)$ group associated to the weights $P=(\underline{ \pm, \pm, \ldots 0})$ appears (underlining meaning permutation over the 16 entries) and the corresponding 16 Cartan oscillators. Therefore, at this moduli point, the enhanced gauge group is $\mathrm{SO}(32)_{L} \times \mathrm{SU}(2)_{L} \times \mathrm{U}(1)_{R}$. In the notation of $(2.19)$ this full set of massless states corresponds to $\check{\mathbb{P}}_{\mathrm{SU}(2) \times \mathrm{SO}(32) \times \mathrm{U}(1)}=( \pm 1, \pm 1 ; 0, \ldots ; 0),(0,0 ; \pm, \pm, \ldots 0)$.

We can break the enhanced gauge group $\mathrm{SO}(32)_{L} \times \mathrm{SU}(2)_{L} \times \mathrm{U}(1)_{R}$ to $\mathrm{SO}(32)_{L} \times$ $\mathrm{U}(1)_{L} \times \mathrm{U}(1)_{R}$ or to $\mathrm{U}(1)_{L}^{17} \times \mathrm{U}(1)_{R}$ depending on the direction of the moduli space on which we move.

For instance, by sliding away from the self-dual radius, charged $\mathrm{SU}(2)$ vectors become massive with mass square $m_{-}^{2}$ with $m_{-}=\sqrt{\frac{2}{\alpha^{\prime}}} a_{-}=\frac{1}{R}-\frac{1}{\tilde{R}}$, where $a_{ \pm}$are defined in (A.16). The algebra (2.19) becomes

$$
\begin{aligned}
{\left[E_{+}, E_{-}\right] } & =2\left(a_{+} H_{3}+a_{-} H_{\overline{3}}\right) & {\left[E_{P}, E_{-P}\right] } & =P_{I} H_{I} \\
{\left[H_{3}, E_{ \pm}\right] } & = \pm a_{+} E_{ \pm} & {\left[H_{I}, E_{P}\right] } & =P_{I} E_{P} \\
{\left[H_{\overline{3}}, E_{ \pm}\right] } & = \pm a_{-} E_{ \pm} & {\left[E_{P_{1}}, E_{P_{2}}\right] } & =f_{P_{1} P_{2} P_{3}} E_{P_{3}} .
\end{aligned}
$$

The subindices \pm denote the two roots of $\mathrm{SU}(2)$, the subindex 3 denotes the corresponding Cartan whereas $f_{P_{1} P_{2} P_{3}}$ are the structure constants of $\mathrm{SO}(32)$ where $P_{I}$ are the roots and $H_{I}$ the Cartan generators. At the self-dual radius we have $a_{-}=0, a_{+}=1$ and the $\mathrm{SU}(2)$ gauge algebra is recovered. 
By turning on Wilson lines $A^{I}$ the group is broken to $\mathrm{U}(1)_{L}^{17} \times \mathrm{U}(1)_{R}$. The algebra becomes

$$
\begin{aligned}
{\left[E_{+}, E_{-}\right] } & =\left(2-\frac{1}{2} A^{2}\right) H_{3}+\left(\frac{1}{2} A^{2}\right) H_{\overline{3}}+A^{I} H_{I} & {\left[E_{P}, E_{-P}\right] } & =P_{I} H_{I}-(P \cdot A) H_{3}-(P \cdot A) H_{\overline{3}} \\
{\left[H_{3}, E_{ \pm}\right] } & = \pm\left(2-\frac{1}{2} A^{2}\right) E_{ \pm} & {\left[H_{I}, E_{P}\right] } & =P_{I} E_{P} \\
{\left[H_{\overline{3}}, E_{ \pm}\right] } & = \pm\left(\frac{1}{2} A^{2}\right) E_{ \pm} & {\left[E_{P_{1}}, E_{P_{2}}\right] } & =f_{P_{1} P_{2} P_{3}} E_{P_{3}} \\
{\left[H_{I}, E_{ \pm}\right] } & = \pm A^{I} E_{ \pm} & {\left[H_{\overline{3}}, E_{P}\right] } & =-(P \cdot A) E_{P} .
\end{aligned}
$$

As discussed, the vector boson masses are identified with the structure constants mixing Left and Right indices. Therefore we find that $\mathrm{SU}(2)$ charged vectors $A_{\mu}^{ \pm}$acquire a mass $m_{\mathrm{SU}(2)}=\left|f_{\overline{3}_{ \pm}}{ }^{ \pm}\right|=\frac{1}{2} A^{2}$ whereas $\mathrm{SO}(32)$ charged vectors masses are $m_{\mathrm{SO}(32)}=\left|f_{\overline{3} P-P}\right|=|P \cdot A|$. As discussed in the general case, the above commutators satisfy Jacobi Identities and define an $\mathrm{SU}(2) \times \mathrm{SO}(32)$ algebra now involving massive states. Let us recall that from DFT perspective the algebra is obtained through generalized Lie derivatives of the twists $E_{A}(\mathbb{Y})$. The explicit twist for the $\mathrm{SU}(2)$ sector is given in (A.15).

\subsubsection{SO(34)}

Other enhanced groups can be obtained at different points in moduli space. points in moduli space can lead to different enhancements. For instance, by choosing $[4,5]$ $\tilde{R}=\sqrt{2 \alpha^{\prime}}$ and $R A=(-1, \ldots 0)$ we notice that for $\tilde{p}=0$ massless states are obtained if $P=( \pm, \pm, \ldots 0)$, namely an $\mathrm{SO}(32)$ root, if KK momenta $p=-P^{1}$ is selected. Moreover, the $\mathrm{SO}(32)$ weights $P=( \pm, \pm, \ldots 0),(0, \ldots 0),(2, \ldots 0)$ combined with $\tilde{p}= \pm 1$ lead to $l_{L}=( \pm ; \pm, \ldots 0)$ states that combined with the $\mathrm{SO}(32)$ roots lead to massless states with charged operators associated to $l_{L}=( \pm ; \pm \ldots 0)$ corresponding to the well known $\operatorname{SO}(34)$ enhanced group $[4,5]$.

Recall thet our description holds at the neighborhood of the $\mathrm{SU}(2) \times \mathrm{SO}(32) \times \mathrm{U}(1)$ point (defined by a specific choice of generalized momentum $\check{\mathbb{P}}_{\mathrm{SU}(2) \times \mathrm{SO}(32) \times \mathrm{U}(1)}$ and moduli fields) or $\mathrm{SO}(34)$ point with different generalized momenta $\breve{\mathbb{P}}_{\mathrm{SO}(34)}$ and moduli fields but it is not possible to continuously interpolete between both points.

\section{Including fermions}

The action (2.3), for $d=4$, is nothing but the $N=4$ bosonic (electric) sector of a generic gauged supergravity theory (see for instance $[18-20,25,26]$ ). We then see that, ScherkSchwarz reduction of DFT provides a way of deriving this gauged supergravity sector.

Inclusion of the magnetic sector requires considering EFT (see for instance [27, 28]) or an extension of the initial global group. The inclusion of fermions from a DFT point of view was considered in several works $[29,30]$ and, in particular, a Scherk- Schwarz like reduction was proposed in [32] in the context of the superstring.

The aim of the present section is to show that the mechanism of gauge symmetry enhancing- breaking through moduli dependent fluxes, found for the bosonic sector, is reproduced in the fermionic sector. 
By invoking supersymmetry we conclude that the fermionic sector is just the fermionic sector of gauged supergravities discussed in the literature. We first concentrate in the $N=4$ case in four dimensions and discuss its generalization later on. Therefore, we must deal with the global symmetry group $O(6+n, 6)$. In particular we concentrate in the fermionic mass terms. For instance, quadratic terms containing the gravitini $\psi_{\mu i}$ and gaugini $\lambda_{j}^{a}$ read $[18,25,26]$

$$
e^{-1} \mathcal{L}_{\text {f.mass }}=\frac{1}{3} g A_{1}^{i j} \bar{\psi}_{\mu i} \Gamma^{\mu \nu} \psi_{\mu j}+i g A_{2 a i}{ }^{j} \bar{\psi}_{\mu i} \Gamma^{\mu} \lambda_{j}^{a}+A_{3 a b}{ }^{i j} \bar{\lambda}_{j}^{a} \lambda_{j}^{a}+\text { h.c. },
$$

where the matrices $A_{1}^{i j}, A_{2 a i}{ }^{j}, A_{3 a b}{ }^{i j}$ are known as shift matrices. Here indices $i$ span the spinorial representation of $\mathrm{SO}(6)$ or, equivalently, the 4-dimensional representation of $\mathrm{SU}(4)$, the universal cover of $\mathrm{SO}(6)$. $\mathrm{SO}(6)$ vectors $v_{\hat{m}}$ can be recast in terms of the antisymmetric combinations of spinorial representations, or, equivalently in terms of antisymmetric $\mathrm{SU}(4)$ six dimensional representation $v^{i j}$ through $v_{\hat{m}}\left(\gamma^{\hat{m}}\right)^{i j}=v^{i j}$ where

$$
v^{i j}=v^{[i j]} \quad \text { and } \quad v_{i j}=\left(v^{i j}\right)^{*}=\frac{1}{2} \epsilon_{i j k l} v^{k l} .
$$

The shift matrices are known to depend on scalars through the coset representatives $\mathcal{U}_{A}{ }^{\bar{A}}(x)$ defining the scalar matrix (A.11). For internal indices such matrix reads

$$
\mathcal{H}_{A B}(x)=\delta_{\bar{A} \bar{B}} \mathcal{U}_{A}{ }^{\bar{A}} \mathcal{U}_{B}{ }^{\bar{B}},
$$

with

$$
\mathcal{U}_{A}{ }^{\bar{A}}(x) \equiv\left(\mathcal{U}_{A}^{a} ; \mathcal{U}_{A}^{\hat{I}}\right)=\left(\mathcal{U}_{A}^{a} ; \mathcal{U}_{A}^{i j}\right)
$$

and where the $\mathrm{SO}(6)$ vector index $\hat{I}$ was expressed in terms of the spinor indices $i j$ in the last term.

The shift matrices then read (see for instance $[18,25,26]$ )

$$
\begin{aligned}
& \text { gravitini-gravitini : } \quad A_{1}^{i j} \propto\left(\mathcal{U}_{A}{ }^{k l}\right)^{*} \mathcal{U}_{B}{ }^{i k} \mathcal{U}_{C}{ }^{j l} f^{A B C} \\
& \text { gravitini-gaugini : } \quad A_{2 a i}{ }^{j} \propto \mathcal{U}_{A}{ }^{a}\left(\mathcal{U}_{B}{ }^{i k}\right)^{*} \mathcal{U}_{C}{ }^{j k} f^{A B C} \\
& \text { gaugini-gaugini : } A_{3 a b}{ }^{i j} \propto \mathcal{U}_{A}{ }^{a} \mathcal{U}_{B}{ }^{b} \mathcal{U}_{C}{ }^{i j} f^{A B C},
\end{aligned}
$$

where we have used $f^{A B C}$ to denote the electric sector gaugings $f_{+}{ }^{A B C}$, the + subindex indicating the electric sector [18]. In order to read vector masses we need to keep the constant term in the expansion of $\mathcal{U}_{A}{ }^{\bar{A}}$ in scalar fluctuations (see (3.3)) reproducing the metric expansion $\mathcal{H}_{A B}=\delta_{A B}+\mathcal{O}(M)$. Therefore $\left(\mathcal{U}_{A}^{b} ; \mathcal{U}_{A} \hat{I}\right)=\left(\delta_{A}{ }^{b} ; \delta_{A} \hat{I}\right)$ with

$$
\mathcal{U}_{A}^{i j}=\delta_{A \hat{m}}\left(\gamma^{\hat{m}}\right)^{i j} .
$$

By replacing this expansion into shift matrices expressions we find

$$
\begin{aligned}
A_{1}^{i j} & \propto \delta_{A, \hat{a}}\left(\gamma^{\hat{a}}\right)^{* k l} \delta_{B, \hat{I}}\left(\gamma^{\hat{I}}\right)^{i k} \delta_{C, \hat{c}}\left(\gamma^{\hat{c}}\right)^{j l} f^{A B C}=\left(\gamma^{\hat{a}}\right)^{* k l}\left(\gamma^{\hat{I}}\right)^{i k}\left(\gamma^{\hat{c}}\right)^{j l} f^{\hat{a} \hat{I} \hat{c}} \\
A_{2 a i}{ }^{j} & \propto\left(\gamma^{\hat{I}}\right)^{* i k}\left(\gamma^{\hat{c}}\right)^{j k} f^{a \hat{I} \hat{c}} \\
A_{3 a b}{ }^{i j} & \propto\left(\gamma^{\hat{c}}\right)^{i j} f^{a b \hat{c}} .
\end{aligned}
$$


By identifying the gaugings $f^{A B C}$ with the fluxes defined above and by using that fluxes involving more than one Right index vanish $\left(f^{\hat{a} \hat{I} \hat{c}}=f^{a \hat{I} \hat{c}}=0\right)$ we find that, gravitini remain massless, as expected (same is valid for dilatini). On the other hand, gaugini masses are proportional to $f^{a b c \hat{c}}$, so having the same masses as their vector boson super-partners, vanishing at the self-dual enhancing points. Together with scalars and vector bosons they fill up the $\mathcal{N}=4$ vector supermultiplet multiplet.

Let us argue that the discussion presented here for $d=4$ extends to other dimensions. In fact, for half-maximal theories, the scalars form a coset $G / H=\operatorname{SO}(d, d+n) / \operatorname{SO}(d) \times$ $\mathrm{SO}(d+n)$ and are encoded in a coset representative $\mathcal{U}_{A}^{\bar{A}}=\left(\mathcal{U}_{A}{ }^{a} ; \mathcal{U}_{A}{ }^{\hat{I}}\right)$ as in (3.4) where $A$ is now a $G=\mathrm{SO}(d, d+n)$-vector index, $a$ is a $\mathrm{SO}(d)$-vector index and $\hat{I}$ is a $\mathrm{SO}(d+n)$-vector index. The index $\hat{I}$ is expressed in terms of spinor indices since fermions transform under $H=\operatorname{Spin}(d) \times \mathrm{SO}(d+n)$. From the full set of possible gaugings it is still possible to choose a subset parametrized by an antisymmetric $G$-tensor, namely, $f_{A B C}$. For instance, in $d=4$ the full set of gaugings is parametrized by $\xi_{\alpha A}$ and $f_{\alpha A B C}(\alpha= \pm$ is the electri-magnetic index) and we have restricted to $\xi_{\alpha A}=0$ and $f_{+A B C}=f_{A B C}, f_{-A B C}=0$. The same applies in other dimensions. ${ }^{4}$ The fermion shift matrices would couple to scalars through the embedding tensor and therefore they will necessarily have the same form as in (3.5) but where $i, j$ indices span the spinorial representation of $\operatorname{Spin}(d)$. The reader should be aware that the actual mass terms of bilinear fermions are linear combinations of the above terms including scalars factors.

As before when the gaugings are the ones coming from an enhancement point of the string moduli space the structure constant (the fluxes) will take the values of the previous section. In these points, the gravitini shift matrices are zero and supersymmetry is preserved. Away from the point of enhancement scalars vectors and fermions organized into a massive supermultiplet.

\section{Summary and outlook}

In the present work we have shown how DFT can provide an interesting description of the gauge symmetry enhancing-breaking process that occurs in the heterotic strings at specific points of moduli space. The construction relies on previous ideas used to describe this process in the bosonic string case. The three key ingredients encoding enhancing information are: a global $O\left(n_{1}, n_{2}\right)$ invariant gauged (super) gravity action, a scalar fluctuation expansion of a generalized scalar metric and the presence of generalized, moduli dependent, 3-form fluxes.

The heterotic effective action is obtained by choosing the global group $O(n, r)$ (where $n=\operatorname{dim} G_{L}$ is the dimension of the enhanced group at the fixed point and $r=10-d$ the number of compact dimensions) and by identifying 3-form fluxes $f_{A B C}(\Phi)$ with the internal momenta of the string. Recall that indices are conveniently written as $A=(a, \hat{I})$ with $a=1, \ldots n, \hat{I}=1, \ldots r$. At a point of enhancement $\Phi_{0}$ the only non vanishing fluxes are those with only Left indices $f_{a b c}\left(\Phi_{0}\right)$ reproducing the structure constants of the $G_{L}$

\footnotetext{
${ }^{4}$ If we wanted it to also hold in $d=9$ and $d=8$ we should necessarily include vector multiplets, thus, $n \geq 1$. Otherwise, $f_{A B C}=0$, see $[25,26,34]$.
} 
group. Away from this point, mixed indices give rise to non-vanishing fluxes $f_{a b \hat{I}}(\Phi)$. These mixed indices fluxes govern the vector boson masses, the scalar masses and the structure of the would be Goldstone bosons. It is worth emphasizing that this structure exactly matches the string theory results with the correct full dependence on moduli fields.

By invoking supersymmetry, a fermionic sector can also be included. In particular we have shown that moving away from the enhancing point $\Phi_{0}$ produces the expected masses for gaugini partners of massive vector bosons while keeping supersymmetry unbroken.

Let us address some open questions. In DFT the generalized fluxes appear as generalized Lie derivatives, involving internal coordinates $\mathbb{Y}^{M}$, of generalized internal frame vectors $E_{A}(\mathbb{Y})$. In a generic construction, the internal coordinates transform in the vector representation of the global group $O\left(n_{1}, n_{2}\right)$, namely $M=1, \ldots, n_{1}+n_{2}$ and the same is valid for the frame index $A$. However, it appears that in order to reproduce the above fluxes, just a dependence on the "true" internal Left and Right $16+r+r=36-2 d$ coordinates, associated to string coordinates would be needed. In fact this was shown to be the case for some specific examples in [1,3] (see also [2]) for the bosonic string case. In a similar line of reasoning a dependence on $\mathbb{Y}=\left(Y^{I}, y_{L}^{I}, y_{R}^{\hat{I}}\right)$ with $I=1, \ldots 16 ; \hat{I}=1, \ldots r$ would be expected. Therefore, the tangent space here, spanned by $A$ would account for the gauge symmetry enhancement, associated to states with non vanishing KK momenta and windings, but the "physical space" would be the string torus (including $\Gamma_{16}$ ). The explicit construction for the heterotic string here remains as an open question.

Recall that our description is valid close to a given moduli point. When moving from one point of enhancement to a new point the dimension of the gauge group can drastically change and, therefore, the dimension of the tangent space. Even if, as stressed in [3], these tangent directions are not physical dimensions an explanation of how, moving continuously from one point of enhancement to another could lead to a discrete change in the number of these extra tangent dimensions is still lacking. DFT description would presumably require the introduction of extra states, mimicking the string theory situation. Following the suggestions in [3] this could be presumably achieved by considering a sort of generalized KK expansion on generalized momenta $\mathbb{L}$ of the different fields coming into play. Thus, very schematically a vector boson corresponding to a charged generator would $\operatorname{read}^{5}$

$$
A_{L \nu}(x, \mathbb{Y})=\sum_{\mathbb{L}} A_{L \nu}^{(\mathbb{L})}(x) e^{i \mathbb{L}_{M} \mathbb{Y}^{M}} \delta\left(\mathbb{L}^{2}, 1\right)=\sum_{\mathbb{L}} A_{L \nu}^{(\mathbb{L})}(x) e^{i K \cdot Y+i k_{L} \cdot y_{L}+i k_{R} \cdot y_{R}} \delta\left(\mathbb{L}^{2}, 1\right),
$$

where $K^{I}, k_{L}^{m}, k_{R}^{m}$ are functions of the moduli. Therefore, when moving continuously along the moduli space, and for specific values of generalized momenta $\mathbb{L}$ in above sum, $k_{R}=0$ and the associated vector fields $A_{L \nu}^{(\mathbb{L})}(x)$ become massless. The neighborhood of each of such points is what our description would be capturing.

In order to address the description of the enhancement process we made a specific choice of generalized fluxes $f_{A B C}$ with $A=(a, \hat{I})$ by keeping just the indices leading to the enhanced gauge group structure constants at the enhancing moduli point and setting the other components to zero. However, it appears interesting to explore the meaning of other

\footnotetext{
${ }^{5}$ Similar expansions were considered in [41] for the bosonic string case and for $\mathbb{L}^{2}=0$.
} 
possible components. In fact, we have already mentioned that, if we look at all indices running from $1, \ldots r+n$, namely $a=i, \hat{I}=1, \ldots r$ the corresponding fluxes encode the geometric and non-geometric (closed string) fluxes discussed in the literature [22-24]. In the six dimensional case, these fluxes span the $\mathbf{2 2 0}$ representation of $O(6,6)$. Interestingly enough, the quadratic constraints (2.8) mixing these fluxes with the gauge group ones would impose restrictions on the possible gauge groups. This is reminiscent of the FreedWitten anomaly [38] cancellation requirements discussed in [39], in the context of Type II string, where such conditions where obtained from quadratic constraints. Such mixings, in the heterotic string Abelian case, were found also in [40]. Notice that there are still other fluxes components to be considered that, in the context of Type II would correspond to mixings of open string indices with closed string ones. Heterotic/Type I duality could shed light on their possible interpretation.

\section{Acknowledgments}

We thank D. Marqués, C. Nuñez and A. Rosabal for useful discussions. This work was supported by CONICET grant PIP-11220110100005 and PICT-2012-513. G.A. thanks the Instituto de Física Teórica (IFT UAM-CSIC) in Madrid for its support via the Centro de Excelencia Severo Ochoa Program under Grant SEV-2012-0249.

\section{A Heterotic DFT}

In this section we briefly present some basic ingredients of DFT in the so called dynamical fluxes formulation. Details can be found in the references [10, 11, 15-17, 33].

In this formulation, the field degrees of freedom (metric, antisymmetric field, 1-forms) are encoded into generalized frame vectors $E_{\overline{\mathcal{A}}} \mathcal{M}^{\mathcal{M}}$ that parametrize a coset $\mathrm{G} / \mathrm{H}$ where $G$ is a duality group. Generalized metric is thus obtained from

$$
H=E_{\overline{\mathcal{A}}} S^{\overline{\mathcal{A}} \overline{\mathcal{B}}} E_{\overline{\mathcal{B}}}
$$

where the $S_{\overline{\mathcal{A}} \overline{\mathcal{B}}}$ is given by

$$
S_{\overline{\mathcal{A}} \overline{\mathcal{B}}}=\left(\begin{array}{cc}
s^{\bar{a} \bar{b}} & 0 \\
0 & s_{\bar{a} \bar{b}}
\end{array}\right),
$$

and $s_{\bar{a} \bar{b}}$ is the $d$-dimensional Minkowski metric.

A scalar field $d$ incorporates the dilaton. Gauge invariance appears through a generalized Lie derivative $\mathcal{L}_{\xi}$. Transformations under this derivative lead to the dynamical fluxes.

The DFT action reads

$$
S=\int d X e^{-2 d} \mathbb{R}
$$

with

$$
\begin{aligned}
\mathbb{R}= & \mathcal{F}_{\overline{\mathcal{A}} \overline{\mathcal{B}} \overline{\mathcal{C}}} \mathcal{F}_{\overline{\mathcal{D}} \bar{E} \bar{F}}\left[\frac{1}{4} S^{\overline{\mathcal{A}} \overline{\mathcal{D}}} \eta^{\overline{\mathcal{B}} \bar{E}} \eta^{\bar{C} \bar{F}}-\frac{1}{12} S^{\overline{\mathcal{A}} \overline{\mathcal{D}}} S^{\overline{\mathcal{B}} \overline{\mathcal{E}}} S^{\overline{\mathcal{C}} \overline{\mathcal{F}}}-\frac{1}{6} \eta^{\overline{\mathcal{A}} \overline{\mathcal{D}}} \eta^{\overline{\mathcal{B}} \overline{\mathcal{E}}} \eta^{\overline{\mathcal{C}} \overline{\mathcal{F}}}\right] \\
& +\mathcal{F}_{\overline{\mathcal{A}}} \mathcal{F}_{\overline{\mathcal{B}}}\left[\eta^{\overline{\mathcal{A}} \overline{\mathcal{B}}}-S^{\overline{\mathcal{A}} \overline{\mathcal{B}}}\right]
\end{aligned}
$$


where the dynamical fluxes $\mathcal{F}_{\overline{\mathcal{A}} \overline{\mathcal{B}} \overline{\mathcal{C}}}$ and $\mathcal{F}_{\overline{\mathcal{A}}}$ are defined in terms of the generalized Lie derivative $\mathcal{L}$ and vielbeins $E_{\overline{\mathcal{A}}}$ by

$$
\begin{aligned}
\mathcal{F}_{\overline{\mathcal{A}} \overline{\mathcal{B}} \overline{\mathcal{C}}} & =E_{\overline{\mathcal{C}} \mathcal{M}} \mathcal{L}_{E_{\overline{\mathcal{A}}}} E_{\overline{\mathcal{B}}} \mathcal{M} \\
\mathcal{F}_{\overline{\mathcal{A}}} & =-e^{2 d} \mathcal{L}_{E_{\overline{\mathcal{A}}}} e^{-2 d} .
\end{aligned}
$$

The field $d$ incorporates the dilaton field and transforms like a measure

$$
\mathcal{L}_{V} e^{-2 d}=\partial_{P}\left(V^{\mathcal{P}} e^{-2 d}\right) .
$$

The indices $\mathcal{M}$ take values in the fundamental representation of the group $G$ whereas the flat indices $\overline{\mathcal{A}}$ run over $H$. Usually, motivated by the bosonic string construction the group $G$ is chosen to be $O(d, d)$ with $d$ the space-time dimensions. However the construction is more general and we can choose $G=O\left(d_{L}, d_{R}\right)$ generically containing $O(d, d)$ and $H=O\left(d_{L}\right) \times O\left(d_{R}\right)$. Indices are raised and lowered with a symmetric metric that can be chosen as

$$
\eta^{P Q}=\left(\begin{array}{cc}
1_{d_{L}} & 0 \\
0 & -1_{d_{R}}
\end{array}\right) .
$$

A numerically similar matrix $\eta^{\overline{\mathcal{A}} \overline{\mathcal{B}}}$ is used for flat indices.

The dynamical fluxes depend on the generic coordinates spanning a vector representation of $O\left(d_{L}, d_{R}\right)$. The above action is generically non invariant under generalized diffeomorphisms generated by the generalized Lie derivative unless some constraints are imposed. A consistent solution is given by a generalized Scherk Schwarz reduction where the frame is split into a space-time dependent part and an internal one $[8,9,15,16]$. The Lie derivative becoming a gauge transformation plus usual space-time diffeomorphisms.

Since we are interested in the description of the heterotic case we perform a specif choice suitable for its description (see also [15, 27, 28, 35-37]). Inspired by the coset structure presented in (2.2) we choose $d_{L}=d+n_{c}+26-d=d+n$ and $d_{R}=d+10-d=d+r$.

We also choose the fields to depend on the coordinates $\mathbb{X}^{\mathcal{M}}=\left(x_{\mu}, \tilde{x}^{\mu}, \mathbb{Y}^{M}\right)$ where $\mathbb{Y}^{M}=\left(y_{L}^{m_{L}}, y_{R}^{m_{R}}\right) . \quad M=\left(m_{L}, m_{R}\right)$ is an internal index with $m_{L}=1, \ldots, n$ and $m_{R}=1, \ldots, 10-d$ whereas $\mu=1, \ldots, d$. Here $r=10-d$ is the number of compact dimensions and $n=n_{c}+26-d$ is the number of extra directions needed to achieve the enhancement. Note that in section 2 the index $M$ is denoted as $A$ and $m_{L}=a, m_{R}=\hat{I}$. As usual, $\tilde{x}^{\mu}$ coordinates are just an artifact and can be dropped away or, in the DFT language, the strong constraint must be used on the space-time part. Explicitly the Scherk-Schwarz reduction ansatz reads

$$
E_{\overline{\mathcal{A}}}\left(x, y_{L}, y_{R}\right)=\mathcal{U}_{\overline{\mathcal{A}}}{ }^{\mathcal{A}}(x) E_{\mathcal{A}}^{\prime}\left(y_{L}, y_{R}\right)
$$

The matrix $\mathcal{U}$ encodes the field content in the effective theory, while $E^{\prime}$ is a generalized twist that depends on the internal coordinates. All the dependence on the internal coordinates occurs through this twist.

By introducing the splitting ansatz (A.9) into the expression (A.1) for the generalized metric we can write

$$
H=S^{\overline{\mathcal{A}} \overline{\mathcal{B}}} \mathcal{U}_{\overline{\mathcal{A}}}{ }^{\mathcal{A}}(x) E_{\mathcal{A}}^{\prime}(\mathbb{Y}) \mathcal{U}_{\overline{\mathcal{B}}}^{\mathcal{B}}(x) E_{\mathcal{B}}^{\prime}(\mathbb{Y})=\mathcal{H}^{\mathcal{A B}}(x) E_{\mathcal{A}}^{\prime}(\mathbb{Y}) E_{\mathcal{B}}^{\prime}(\mathbb{Y})
$$


where all the field dependence on space-time coordinates is encoded in

$$
\mathcal{H}^{\mathcal{A B}}(x)=S^{\overline{\mathcal{A}} \overline{\mathcal{B}}} \mathcal{U}_{\overline{\mathcal{A}}} \mathcal{A}^{\mathcal{A}}(x) \mathcal{U}_{\overline{\mathcal{B}}}{ }^{\mathcal{B}}(x)
$$

In particular, when the indices take internal values $A, B=1, \ldots, n+r$, the matrix of $\mathcal{H}^{A B}$ parametrizes the scalar content of the theory.

By restricting the expression for the generalized Lie derivative to the specific case of the twist it is found that

$$
\begin{aligned}
\mathcal{L}_{E_{A}^{\prime}} E_{B}^{\prime} & =\frac{1}{2}\left[E_{A}^{\prime P} \partial_{P} E_{B}^{\prime M}-E_{B}^{\prime P} \partial_{P} E_{A}^{\prime M}+\eta^{M N} \eta_{P Q} \partial_{N} E_{A}^{\prime P} E_{B}^{\prime Q}\right] \\
{\left[E_{A}^{\prime}, E_{B}^{\prime}\right] } & =\mathcal{L}_{E_{A}^{\prime}} E_{B}^{\prime}=f_{A B}{ }^{K} E_{K}^{\prime},
\end{aligned}
$$

where here all indices are internal. The fluxes $f_{A B}{ }^{K}$ of the generalised Scherk-Schwarz reduction must be constants and must satisfy the constraints

$$
f_{A B C} \equiv \eta_{C K} f_{A B}{ }^{K}=f_{[A B C]}, \quad f_{[A B}{ }^{K} f_{C D] K}=0
$$

in order for the algebra to close.

When replacing above results into the initial DFT action (A.3), the expression for the gauged DFT action (2.3) is obtained.

Let us stress that a specific selection of values for the fluxes $f_{A B C}$, constructed out from the internal frame derivatives (A.12) will be associated to a specific dependence on the generalized coordinates. For instance, for the extreme case of a coordinate independent frame leads to an abelian compactification, and corresponds to a KK reduction, for instance.

In particular, it was shown in [1-3] that, at least for some cases, for the twists $E_{A}$ to reproduce the structure constants of the gauge group the twist only depends on the true internal coordinates of the torus. Thus, for a circle compactification of the bosonic string the twist only depends on the circle coordinate $y_{L}$ and its dual $y_{R}$. Explicitly [3],

$$
\begin{aligned}
E_{ \pm} & =i \sqrt{\alpha^{\prime}}\left(e^{\mp i w / \sqrt{\alpha^{\prime}}}, \pm i e^{\mp i w / \sqrt{\alpha^{\prime}}}, 0 ; 0\right) \quad E_{3}=-i \sqrt{\alpha^{\prime}}(0,0,1 ; 0) \\
\bar{E}_{\hat{3}} & =-i \sqrt{\alpha^{\prime}}(0,0,0 ; 1)
\end{aligned}
$$

where $w=a_{+} y_{L}+a_{-} y_{R}, \bar{w}=a_{-} y_{L}+a_{+} y_{R}$ and

$$
a_{\mp}=\sqrt{\frac{\alpha^{\prime}}{2}}\left[\frac{1}{R} \mp \frac{1}{\tilde{R}}\right]
$$

It is easy to check that, by inserting this twist expression into (A.12) and noticing that the only contributions to the derivatives come from $\partial_{A}=\left(0,0, \partial_{y_{L}} ; \partial_{y_{R}}\right)$, the $\mathrm{SU}(2)$ algebra is reproduced. Here we just assume that there exists a choice of internal coordinates such that (A.13) leads to the desired gauge group structure constants and leave the construction of the explicit twist for future work. 


\section{B Some heterotic string basics}

We summarize here some string theory ingredients (that can be found in string books) needed in the body of the article. We mainly concentrate in the $\mathrm{SO}(32)$ string.

For a heterotic string compactified to $d$ space-time dimensions, Left and Right momenta are encoded in momentum

$$
\mathbb{L}=\left(l_{L}, l_{R}\right)
$$

defined on a self-dual lattice $\Gamma_{26-d, 10-d}$ of signature $(26-d, 10-d)$. By writing $l_{L}^{\check{I}}=\left(K_{L}^{I}, k_{L, m}\right)$ with $I=1, \ldots, 16$ and $m=1, \ldots 10-d=r$, the moduli dependent momenta, read

$$
\begin{aligned}
K_{L}^{I} & =P^{I}+R A_{n}^{I} \tilde{p}^{n} \\
k_{L, m} & =\sqrt{\frac{\alpha^{\prime}}{2}}\left[\frac{p_{m}}{R}+\left(g_{m n}-B_{m n}\right) \frac{\tilde{p}^{n}}{\tilde{R}}-P^{I} A_{m}^{I}-\frac{R}{2} A_{m}^{I} A_{n}^{I} \tilde{p}^{n}\right] \\
k_{R, m} & =\sqrt{\frac{\alpha^{\prime}}{2}}\left[\frac{p_{m}}{R}+\left(-g_{m n}-B_{m n}\right) \frac{\tilde{p}^{n}}{\tilde{R}}-P^{I} A_{m}^{I}-\frac{R}{2} A_{m}^{I} A_{n}^{I} \tilde{p}^{n}\right],
\end{aligned}
$$

where $g_{m n}, B_{m n}$ are internal metric and antisymmetric tensor components, $A_{m}$ are Wilson lines and $p_{n}$ and $\tilde{p}^{n}$ are integers corresponding to KK momenta and windings, respectively. $P^{I}$ are $\operatorname{Spin}(32)$ weight components.

The mass formulas for string states are

$$
\begin{aligned}
\frac{\alpha^{\prime}}{2} m_{L}^{2} & =\frac{1}{2} l_{L}^{2}+\left(N_{B}-1\right)=\frac{1}{2} K_{L}^{2}+\frac{1}{2} k_{L}^{2}+\left(N_{B}-1\right) \\
\frac{\alpha^{\prime}}{2} m_{R}^{2} & =\frac{1}{2} k_{R}^{2}+\bar{N}_{B}+\bar{N}_{F}+\tilde{E}_{0},
\end{aligned}
$$

where $N, \bar{N}$ are the number of string oscillators, $\tilde{E}_{0}=-\frac{1}{2}(0)$ for NS (R) sector and the level matching condition is $\frac{1}{2} m_{L}^{2}-\frac{1}{2} m_{R}^{2}=0$ or, in terms of above notation

$$
\mathbb{L}^{2}=\frac{1}{2} l_{L}^{2}-\frac{1}{2} k_{R}^{2}=1-N_{B}+\bar{N}_{B}+\bar{N}_{F}+\tilde{E}_{0} .
$$

In particular, massless charged vectors correspond to $\mathbb{L}^{2}=1$. As is well known, there are $10-d+16$ Left gauge bosons corresponding to 16 Cartan generators $\partial_{z} Y^{I} \tilde{\psi}^{\mu}$ of the original gauge algebra as well as $10-d$ KK Left gauge bosons coming from a Left combination of the metric and antisymmetric field $\partial_{z} Y^{i} \tilde{\psi}^{\mu}$. The $10-d$ Right combinations $\partial_{z} X^{\mu} \tilde{\psi}^{m}$ with $m=1, \ldots 10-d$ generate the Right abelian group. These states have $k_{R}=0$ and $l_{L}=0$, with vanishing winding and KK momenta.

Besides these states, a number of different situations arises. At generic points in moduli space $k_{R} \neq 0$ and therefore there are no extra gauge bosons. The gauge group is then $\mathrm{U}(1)_{L}^{26-d} \times \mathrm{U}(1)_{R}^{10-d}$. With Wilson lines turned off, for vanishing winding and momenta and for $P^{2}=2$ the gauge group $\mathrm{SO}(32)_{L} \times \mathrm{U}(1)_{L}^{10-d} \times \mathrm{U}(1)_{R}^{10-d}$ is obtained, as expected from KK reduction of the effective field theory.

For moduli points in $\Gamma_{16}$ such that

$$
k_{R}=0, \quad l_{L}^{2}=2,
$$


an enhancement of the Left gauge symmetry occurs, associated to the presence of massless gauge bosons $e^{i l_{L} \cdot Y_{L}(z)} \tilde{\psi}^{\mu}$. Actually, $l_{L}$ encodes the weight values associated to the charged generators of the enhanced algebra. For specific values of moduli, momenta and windings a maximum enhancement to $\mathrm{SO}(52-2 d)_{L} \times \mathrm{U}(1)_{R}^{10-d}$ gauge group can be obtained. Recall that the rank of the group is always $36-2 d$ and the coset space is

$$
\frac{O(26-d, 10-d, \mathbb{R})}{O(26-d, \mathbb{R}) \times O(10-d, \mathbb{R}) \times O(26-d, 10-d, \mathbb{Z})}
$$

of dimension $(26-d)(10-d)$.

Open Access. This article is distributed under the terms of the Creative Commons Attribution License (CC-BY 4.0), which permits any use, distribution and reproduction in any medium, provided the original author(s) and source are credited.

\section{References}

[1] G. Aldazabal, M. Graña, S. Iguri, M. Mayo, C. Nuñez and J.A. Rosabal, Enhanced gauge symmetry and winding modes in Double Field Theory, JHEP 03 (2016) 093 [arXiv: 1510.07644] [INSPIRE].

[2] Y. Cagnacci, M. Graña, S. Iguri and C. Nuñez, The bosonic string on string-size tori from double field theory, JHEP 06 (2017) 005 [arXiv: 1704.04242] [INSPIRE].

[3] G. Aldazabal, E. Andrés, M. Mayo and J.A. Rosabal, Gauge symmetry enhancing-breaking from a Double Field Theory perspective, JHEP 07 (2017) 045 [arXiv:1704.04427] [INSPIRE].

[4] K.S. Narain, M.H. Sarmadi and E. Witten, A Note on Toroidal Compactification of Heterotic String Theory, Nucl. Phys. B 279 (1987) 369 [INSPIRE].

[5] K.S. Narain, New Heterotic String Theories in Uncompactified Dimensions < 10, Phys. Lett. B 169 (1986) 41 [INSPIRE].

[6] A. Giveon, M. Porrati and E. Rabinovici, Target space duality in string theory, Phys. Rept. 244 (1994) 77 [hep-th/9401139] [INSPIRE].

[7] J. Scherk and J.H. Schwarz, How to Get Masses from Extra Dimensions, Nucl. Phys. B 153 (1979) 61 [INSPIRE].

[8] G. Aldazabal, W. Baron, D. Marqués and C. Nuñez, The effective action of Double Field Theory, JHEP 11 (2011) 052 [Erratum ibid. 11 (2011) 109] [arXiv:1109.0290] [INSPIRE].

[9] D. Geissbuhler, Double Field Theory and $N=4$ Gauged Supergravity, JHEP 11 (2011) 116 [arXiv:1109.4280] [INSPIRE].

[10] W. Siegel, Superspace duality in low-energy superstrings, Phys. Rev. D 48 (1993) 2826 [hep-th/9305073] [INSPIRE].

[11] W. Siegel, Two vierbein formalism for string inspired axionic gravity, Phys. Rev. D 47 (1993) 5453 [hep-th/9302036] [INSPIRE].

[12] C. Hull and B. Zwiebach, Double Field Theory, JHEP 09 (2009) 099 [arXiv:0904.4664] [INSPIRE].

[13] O. Hohm, C. Hull and B. Zwiebach, Background independent action for double field theory, JHEP 07 (2010) 016 [arXiv: 1003.5027] [inSPIRE]. 
[14] O. Hohm, C. Hull and B. Zwiebach, Generalized metric formulation of double field theory, JHEP 08 (2010) 008 [arXiv: 1006.4823] [INSPIRE].

[15] G. Aldazabal, D. Marqués and C. Nuñez, Double Field Theory: A Pedagogical Review, Class. Quant. Grav. 30 (2013) 163001 [arXiv: 1305.1907] [InSPIRE].

[16] M. Graña and D. Marqués, Gauged Double Field Theory, JHEP 04 (2012) 020 [arXiv: 1201.2924] [INSPIRE].

[17] O. Hohm, D. Lüst and B. Zwiebach, The Spacetime of Double Field Theory: Review, Remarks and Outlook, Fortsch. Phys. 61 (2013) 926 [arXiv:1309.2977] [INSPIRE].

[18] J. Schon and M. Weidner, Gauged N=4 supergravities, JHEP 05 (2006) 034 [hep-th/0602024] [INSPIRE].

[19] H. Samtleben, Lectures on Gauged Supergravity and Flux Compactifications, Class. Quant. Grav. 25 (2008) 214002 [arXiv:0808.4076] [INSPIRE].

[20] M. Trigiante, Gauged Supergravities, Phys. Rept. 680 (2017) 1 [arXiv:1609.09745] [INSPIRE].

[21] E. Bergshoeff, I.G. Koh and E. Sezgin, Coupling of Yang-Mills to $N=4, D=4$ Supergravity, Phys. Lett. B 155 (1985) 71 [INSPIRE].

[22] J. Shelton, W. Taylor and B. Wecht, Nongeometric flux compactifications, JHEP 10 (2005) 085 [hep-th/0508133] [INSPIRE].

[23] G. Aldazabal, P.G. Cámara, A. Font and L.E. Ibáñez, More dual fluxes and moduli fixing, JHEP 05 (2006) 070 [hep-th/0602089] [INSPIRE].

[24] G. Aldazabal, E. Andrés, P.G. Cámara and M. Graña, U-dual fluxes and Generalized Geometry, JHEP 11 (2010) 083 [arXiv:1007.5509] [INSPIRE].

[25] E. Bergshoeff, T. de Wit, U. Gran, R. Linares and D. Roest, (Non)Abelian gauged supergravities in nine-dimensions, JHEP 10 (2002) 061 [hep-th/0209205] [INSPIRE].

[26] J.J. Fernandez-Melgarejo, T. Ortín and E. Torrente-Lujan, The general gaugings of maximal $D=9$ supergravity, JHEP 10 (2011) 068 [arXiv:1106.1760] [INSPIRE].

[27] E. Malek, Half-maximal supersymmetry from exceptional field theory, arXiv:1707.00714 [INSPIRE].

[28] E. Malek, From Exceptional Field Theory to Heterotic Double Field Theory via K3, JHEP 03 (2017) 057 [arXiv: 1612.01990] [INSPIRE].

[29] I. Jeon, K. Lee and J.-H. Park, Incorporation of fermions into double field theory, JHEP 11 (2011) 025 [arXiv:1109.2035] [INSPIRE].

[30] I. Jeon, K. Lee and J.-H. Park, Supersymmetric Double Field Theory: Stringy Reformulation of Supergravity, Phys. Rev. D 85 (2012) 081501 [Erratum ibid. D 86 (2012) 089903] [arXiv: 1112.0069] [INSPIRE].

[31] I. Jeon, K. Lee and J.-H. Park, Stringy differential geometry, beyond Riemann, Phys. Rev. D 84 (2011) 044022 [arXiv: 1105.6294] [INSPIRE].

[32] D.S. Berman and K. Lee, Supersymmetry for Gauged Double Field Theory and Generalised Scherk-Schwarz Reductions, Nucl. Phys. B 881 (2014) 369 [arXiv:1305.2747] [INSPIRE].

[33] D. Geissbuhler, D. Marqués, C. Nuñez and V. Penas, Exploring Double Field Theory, JHEP 06 (2013) 101 [arXiv:1304.1472] [INSPIRE]. 
[34] G. Dibitetto, J.J. Fernandez-Melgarejo, D. Marqués and D. Roest, Duality orbits of non-geometric fluxes, Fortsch. Phys. 60 (2012) 1123 [arXiv:1203.6562] [INSPIRE].

[35] O. Hohm and S.K. Kwak, Double Field Theory Formulation of Heterotic Strings, JHEP 06 (2011) 096 [arXiv:1103.2136] [INSPIRE].

[36] O. Hohm, A. Sen and B. Zwiebach, Heterotic Effective Action and Duality Symmetries Revisited, JHEP 02 (2015) 079 [arXiv:1411.5696] [INSPIRE].

[37] O.A. Bedoya, D. Marqués and C. Nuñez, Heterotic $\alpha$ '-corrections in Double Field Theory, JHEP 12 (2014) 074 [arXiv: 1407.0365] [INSPIRE].

[38] D.S. Freed and E. Witten, Anomalies in string theory with D-branes, Asian J. Math. 3 (1999) 819 [hep-th/9907189] [INSPIRE].

[39] G. Aldazabal, P.G. Cámara and J.A. Rosabal, Flux algebra, Bianchi identities and Freed-Witten anomalies in F-theory compactifications, Nucl. Phys. B 814 (2009) 21 [arXiv:0811.2900] [INSPIRE].

[40] N. Kaloper and R.C. Myers, The Odd story of massive supergravity, JHEP 05 (1999) 010 [hep-th/9901045] [INSPIRE].

[41] G. Aldazabal, M. Mayo and C. Nuñez, Probing the String Winding Sector, JHEP 03 (2017) 096 [arXiv: 1611.04927] [INSPIRE]. 